\section{EMBRYARIDDLE \\ Aeronautical University}

SCHOLARLY COMMONS
International Journal of Aviation, Aeronautics, and Aerospace

\title{
Prediction of Gate In Time of Scheduled Flights and Schedule Conformance using Machine Learning-based Algorithms
}

\author{
DEEPUDEV SAHADEVAN \\ National Institute of Technology-Tiruchirappalli, deepudevs@gmail.com \\ Palanisamy $\mathrm{P}$ Dr \\ National Institute of Technology Tiruchirappalli, palan@nitt.edu \\ Varun P. Gopi Dr \\ National Institute of Technology Tiruchirappalli, varun@nitt.edu \\ Manjunath K. Nelli Mr \\ Airports Authority of India, mknelli@aai.aero \\ Asok kumar $\mathrm{K} \mathrm{Mr}$ \\ Airports Authority of India, asokkrishna@aai.aero
}

Follow this and additional works at: https://commons.erau.edu/ijaaa

\section{Scholarly Commons Citation}

SAHADEVAN, D., P, P., Gopi, V. P., Nelli, M. K., \& K, A. (2020). Prediction of Gate In Time of Scheduled Flights and Schedule Conformance using Machine Learning-based Algorithms. International Journal of Aviation, Aeronautics, and Aerospace, 7(4). https://doi.org/10.15394/ijaaa.2020.1521

This Article is brought to you for free and open access by the Journals at Scholarly Commons. It has been accepted for inclusion in International Journal of Aviation, Aeronautics, and Aerospace by an authorized administrator of Scholarly Commons. For more information, please contact commons@erau.edu. 
Over the past several years, the air transportation system has encountered frequent rises in air traffic demand, especially with the introduction of budget airlines. In recent Indian-aviation submits, IATA has presented that by 2026 India is expected to be the third-largest air transport market in the world (International Air Transport Association, 2018) from its current 7th place. Before pandemic COVID-19, increases in air traffic in trend were predicted such that the current air traffic system will not be able to cater the projected air traffic demand of the near future due to the capacity constraints of airport and airspace. In order to overcome this backdrop, there arises a need for the of modernising the air transportation system. Prompt initiatives are being taken by several countries, including India, to develop the future air transport system that will be more robust, predictable, and reliable than today's one. Introduction of Central Air Traffic flow management(CATFM) in India was one of the steps taken towards achieving this objective.

The scope of Air Traffic Flow Management (ATFM), especially in the planning phase, is to balance the air traffic demand with the air system capacity by adopting control options which consist of assigning ground delays to the flights in the initial phase of implementation or alternative routes (rerouting) or both in subsequent phases of implementation. In the last few decades, ATFM has become an active area of interest to the research community. For achieving effective and optimal air traffic flow, predictability is one of the key features airlines try to achieve. Flight operations frequently suffer with mainly four types of delay, which include propagated delays, induced delays, air delays, and random events.

In the aviation industry, where multiple agencies work together, predictability representing a key performance area due to several reasons. Separation of these delays becomes tedious work in realistic contexts, and currently this information is not integrated in the Indian aviation industry, where each stakeholder acts on an independent platform. Improvement in the estimation of such delays or their result would therefore be very supportive of decisionmaking, especially for ATFM traffic management initiatives. As per the International Civil Aviation Organisation (ICAO; Hof, 2005), predictability refers to the ability of the airspace users and ATM service providers to provide consistent and dependable levels of performance. Predictability is essential to airspace users as they develop and operate their schedules. The study investigates the effect of various parameters on the estimation of gate in time and utilises machine learning techniques to enhance predictability.

\section{Problem}

One of the fundamental objectives of ATFM is to balance the air traffic demand and available capacity of an air route, a defined volume of airspace, or an airport. The decisions are taken in the ATFM for air traffic flow control measures of an airport, often influenced by multiple variables, such as the number of 
arrivals per hour(based on flight plans filed), restrictions in air space weather conditions, turn around delay, and delay in trailing legs. A large deviation from filed departure time and flying time will introduce delay across the Air Traffic Network. By accurately calculating estimated landing time and gate in time, unnecessary flight delay to the cost can be reduced. As the deviation of the estimated flying time varies mostly with the actual flying time, the possibility of incorrect assessment of demand is always present. On the other hand, to reduce the effect of delays 'On-time' performance, the airline carriers always 'pad' some additional time in the schedule block time, which leads to inaccurate flow management measures and slot allocation. In addition to that, schedule arrival time variation results in the last minute gate changes which cause inconvenience to the passengers. It is thus important to analyse variables that influence flight block times and use them in the prediction model. In this context, several studies were conducted, some of them has considered flight delay prediction as a regression problem, predicting the delay time, and some others as a classification problem, predicting a time interval where the delay will fall. The problems addressed in this paper are as follows:

1. Improve predictability of Gate to Gate block time.

2. Trace the temporal variation in flying time and improve the predictability of the actual in block time

3. Finally, to improve the predictability of the arrival schedule conformance (Early, On time, and Delay)

\section{Purpose}

The research was conducted to perform focused analysis to determine the cause for traffic congestion in capacity-restricted airports and to determine the impact of non-compliance with the schedule (early or late from the scheduled departure time). Using different machine learning techniques (linear and nonlinear methods), an extension of this research was carried out to trace the predictability of temporal variation in block time. This study facilitates the air traffic flow management decision-making process strengthened by enhancing accuracy of gate-in time estimation using machine learning with minimal features and thereby increase the conformance rate of traffic management initiatives, such as the ground delay method.

\section{Research Questions}

1. How the flight block time (gate to gate) and schedule conformance can be predicted with minimal flight details?

2. Whether the temporal variation in flying time can be better traced using a combination of exponential moving average of flying time and various machine learning methods (regression models)?

3. In order to predict trailing flight delays, how to improve schedule conformance predictability? 


\section{Related Works}

A detailed study about Airline disruptions and the delay was done by Rapajic (2009, 2018). According to him, cutting five minutes of average of 50 per cent of schedules owing to higher predictability, would conserve or make better use of airlines and airport resources, which would be worth some 1,000 million euros per year. In the form of lost revenue, consumer frustration, and potential loss of market share, unpredictability imposes significant costs on airlines. In order to make an impression of greater On-time performance, most of the airlines use schedule padding (adds additional block time) in their schedule, and it may affect airlines actual performance assessment. Hence there is a requirement for adopting an alternative methodology.

According to Donohue et al. (2001), predictability depends on both variability in flight times and arrival rates. The different user experiences benefits of predictability in different way and that will vary according to the service they provide. In their study of predictability provides for any flight operations between Off block time (gate-out) and In Block Time (gate-in) times which includes, flying time, taxi-out and taxi-in movements. The passenger experience was mainly considered in their approach. In our study we mainly focus on how machine learning can be used for better predictability in ATFM decision making. Hoffman et al. (2011) defined predictability as to the reduction of uncertainties in the implementation of ATFM initiatives. Airlines suffer from occurrence of many unplanned events during the flight movement; most of them cannot be foreseen and planned well in advance. So the ATFM initiatives should be communicated clearly in advance as possible and shall be provided to the airport operational provider as well as airlines with time to react.

$\mathrm{Wu}$ (2005) provided an in-depth analysis of problems related to the delay management, optimisation of the operating process and management schedule disruption. However, the model does not consider the effectiveness and contribution of operational variables that impact scheduling. Morrisset and Odoni (2011) compared air traffic delay, runway capacity, scheduling practices, and reliability of flight schedule at 34 major airports in Europe and the United States from 2007 to 2008. Using historical data, proactive management of delay can be done and is directly related to the prediction of arrival time.

Recently researchers have focused towards the classification models to detect reoccurring and period of delays to predict arrival time along with weather information (Choi et al., 2017; Fleurquin et al., 2016; Kim, 2016). Use of machine learning algorithms and methods have lately emerged into aviation and air traffic management research due to data availability and storage capacity. Rebollo et al. (2014) proposed random forest classification and regression algorithms to predict the delays. The predictive models in both delay classification as above or below a given threshold and predict the delay values. Thiagarajan et al. (2017) proposed a 
two-stage predictive model to predict flight delays in departure and arrival, using flight schedule and weather features. Departure delay prediction had comparatively higher error rates due to a weak feature set and the prediction was only confined to delay or no delay. Glina et al. (2012) proposed using Qauntile Regression Forests (QRF; a variant of the Random Forest (RF) that can be used for accurate predictions of aircraft landing times).

Later in Williams (2014), RF was used in real-time diagnose of turbulence associated with thunderstorms, in aviation operations. Followed by a data-driven model Kern et al. (2015), using (RF) method, proposed to predict flight's estimated time of arrival (ETA) with improved accuracy at arrival airports. As Kern et al. showed in their work, the combination of flight data, weather forecasts and airport congestion levels lead to the highest arrival time prediction accuracy. Ding (2017) proposed an MLR model to predict whether a flight will be delayed or not, by considering the problem as a classification with two classes: delayed, for flights with delays above 30 minutes, and non-delayed otherwise. This analysis was carried out in terms of passenger aspect and showed less accuracy in prediction.

In 2016, Choi et al. focused on a relationship between weather, which data from NOAA was collected, and flight delay. As a result, $80.36 \%$ of delay in arrival was predicted by their proposed Random Forest, which is an ensemble learning strategy.

In the air traffic flow management initiative Kuhn (2016) proposed a method for finding similar days. Their study mainly describes a combination of a classification model and a predictive cluster analysis of similar days. Takeichi (2017) proposed optimisation of nominal flight time by estimation/resolution of delay. The possibilities of estimating delay by initial traffic statistics were analysed in their work. Evans et al (2018) introduced a predictor automation tool that allows for route adjustments to be operationally appropriate during a flight and recognizes more efficient airspace routes that are influenced by weather or congestion and better meet airline preferences.

In Brazil's domestic flights with weather data of the same data resource as Thiagarajan et al. (2017) predicted departure and arrival delays using Decision Tree, which uses a tree-like model of decisions and their possible consequences. In Decision Tree classifier's arrival delay, the accuracy is $78 \%$. When the Regression classifier departs delay, the accuracy is $77 \%$. Deepudev et al. (2020) proposed a machine learning based approach for prediction of actual landing time of scheduled flights. This approach uses Exponential Moving average of flying time as one of the feature in Multi Linear Regression (MLR) model. A predictive model of on-time arrival flight using flight data and weather data was proposed by Etani et al (2019). Using the correlation between flight data and weather data on time arrival was classified. Since weather phenomena is highly random in nature, 
the model gives comparatively less predictability with binomial classification only.

\section{Description of the case study data}

New Delhi Indira Gandhi International Airport (ICAO: VIDP, IATA: DEL) has been selected as the study case. It is one among the busiest airports in India, with three parallel runways: $09 / 27,10 / 28$ and 11/29. The average flight movement per day has now crossed 1300, and the total movement was recorded to be 4, 60,424 during the year 2018-19 (Delhi Airport, 2020).

Data collected from different airports using the same excel format and was merged using matching of the date of flying, departure, destination and Call sign. The data consists of general information about the flight under consideration, runway in use, various time milestones, and air traffic information of the destination. The list of variables for time milestones and definitions are listed below:

$\begin{array}{ll}t_{S O}: & \text { Scheduled Off Block Time } \\ t_{A O}: & \text { Actual Off Block Time } \\ t_{A T}: & \text { Actual Take Off Time } \\ t_{A L}: & \text { Actual Landing time } \\ t_{E L}: & \text { Estimated Landing time } \\ t_{S I}: & \text { Scheduled In Block Time } \\ t_{A I}: & \text { Actual In Block Time }\end{array}$

The difference of Actual Off Block Time $\left(t_{A O}\right)$ from the Scheduled Off Block Time $\left(t_{S O}\right)$ gives Minutes of departing late, resulting in negative minutes for flights that has left early. The Flight Length (Flying Time) is calculated by subtracting actual take-off time $\left(t_{A T O}\right)$ from Actual of Landing Time $\left(t_{A L}\right)$. The Gate In - Gate Out(Gate to Gate) Time is calculated by taking the difference of Actual In Block Time $\left(t_{A I}\right)$ and Actual Off Block Time $\left(t_{A O}\right)$, and it is compared with the difference of Scheduled In Block Time $\left(t_{S I}\right)$ and Scheduled Off Block Time $\left(t_{S O}\right)$. The negative values of comparative results (i.e., block time variation; $\left.t_{B T V}\right)$, indicates that the flight took lesser time than scheduled block time.

$$
\begin{aligned}
& \text { ScheduleDelay }(S D)=t_{A O}-t_{S O} \\
& \text { FlyingTime }(F T)=t_{A L}-t_{A T} \\
& \text { BlocktimeVariation }\left(t_{B T V}\right)=\left[t_{A I}-t_{A O}\right]-\left[t_{S I}-t_{S O}\right]
\end{aligned}
$$

The data cleaning performed by removing data with the logically huge duration of flying time and block time, which occurred due to human error during the data collection process. Considering the peak hour period and its matrix 
during the day was more difficult and was different for both departure station and arrival station. Looking at a distribution of scheduled arrival times, it was observed that more than 30 arrival movement per hour was during the day. Statistical analysis of distribution and from our own experience decided that peak hour flights were flights that had Scheduled Departure Times from 02:00 to 06: 00 UTC (07: 30 to 11:30 IST) and 11: 00 to 17: 00 UTC (16: 30 to 22: 30 IST).

In this study, we have considered only the medium category twin-engine operated by scheduled airlines. In this category, we considered the aircraft used by scheduled airlines, which is considered as similar performance. But the study shows that there is minor variation in flying time based on the type of aircraft. The information was gathered from the six airlines used for flights from VABB to VIDP. The six carriers used different type of aircraft made by Boeing (B739, B738, B38M) and Airbus (A319, A320, A32W) which varies is cruising speed and performance that are comparable. Most of the time Air Traffic Controllers (ATC) use the same calculation for their performance and block time.

\section{Preliminary Data Observations}

Initial attempt is to investigate various distributions and to determine outliars for the analysis. Distribution of actual block time vs scheduled arrival time during the day for Mumbai-Delhi city pair is shown in Figure 1. It varies in huge window and this make the prediction of the same very difficult. The same flight on different days uses different block time for the same departure - destination pair. The distribution shows random nature and no specific pattern is followed.

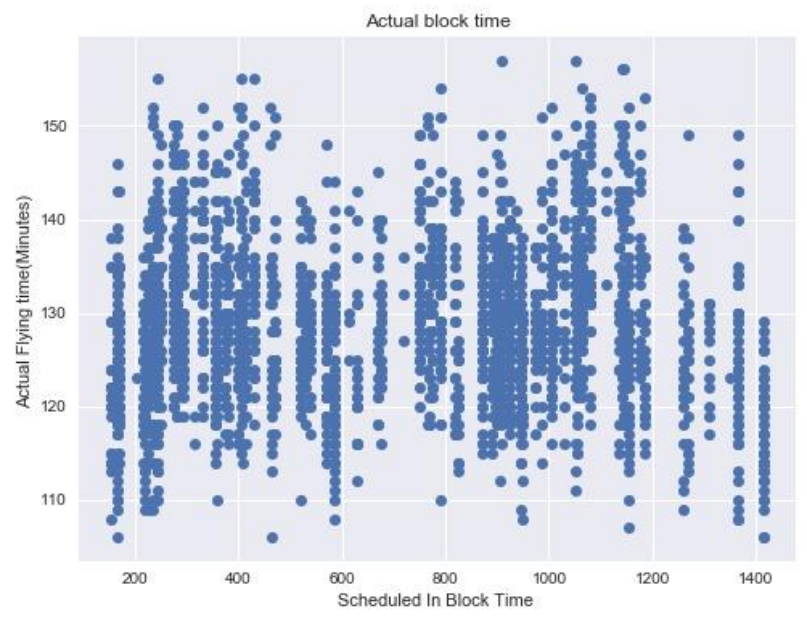

Figure 1. Actual block time distribution during the day.

Using the equation number (1) scheduled departure time was calculated and it was classified as "Early" (Schedule Delay $<=-6$ Minutes), "On Time" (-5 
Minutes $<$ Schedule Delay $<=10$ Minutes) and "Delay" (Schedule Delay is $>10$ Minutes. Initially, 2511 data were considered, and it was observed that more than $65 \%$ of the scheduled flight operated during the trial period have "On-Time" Gate-Out. Further, the distribution of Early departure during the different hours (labelled as Minutes) of the day was analysed.

The mean value $(\mu)$ of flying time was 102.4 Minute, Minimum was 86 Minutes, Maximum was 156 Minutes and standard deviation $(\sigma)$ of 7.5. The data set contained extreme values that are outside the range of what was expected, unlike the other data. These outliers were removed using criteria as more than three-times standard deviations from the mean.

Flyingtime $<=\mu \pm 3 * \sigma$

The data set was limited to flying time of 80 minutes to 125 Minutes. Similarly, Gate out to Gate In time was limited 101 Minutes to 157 Minutes with Mean value of 129.2 minutes. This large window of variation of Flying time and Gate to Gate movement time makes predicting process highly challenging.

Here supervised learning approach is used that interacts with the metadata and provides the data with a label. Regression analysis explores the value of the parameters for the function that best fits an input data set. In this study, we approximated model the function with

1. Regression Tree-M5P

2. Logistic regression

In preliminary data processing, using statistical analysis outliers were determined and the data was cleaned.

\section{Attribute Selection}

Unlike previous works, here prediction was carried out with the minimum number of attributes so that the possibility of overfitting and computational cost can be reduced. The attributes were both numerical as well as nominal/categorical. The numerical attributes include different time milestone during different phases of flight and which were converted to the worth of minutes $(24 * 60)$. The categorical attributes include Day of the week, Runway, call sign, airline and type of aircraft. All the categorical data were converted to binary using One hot encoding Technique. One-Hot Encoding: Categorical data are variables which contain label values in place of numeric values. These variables are often called nominal. Since the model is a regression model, categorical data was converted to a numerical form. For this selection process, pvalue (Dahiru, 2008) or probability value is used, the probability that, when the null hypothesis is true for a given statistical model. The significant level of the pvalue is $<0.05$. The following steps were used for attribute selection: (1) Select significant level of p-value $(<0.05)$ to stay in the model, (2) Fit the model with all 
possible attributes, (3) Check the predictor output with highest $\mathrm{p}-$ value, If $p>$ 0.05 proceed to step 4,else to step 6, (4) Remove the attributes with $p>0.05$, (5) Fit the model without this attribute and proceed to step 3, and (6) Prepare final Model with these attributes.

\section{Proposed Regression Models}

This research work is aimed to predict the actual in block Time $\left(t_{A I}\right)$ of scheduled aircraft using regression analysis. Effect of variation in schedule off Block time (Early/On Time/Delay) for predicting $t_{A I}$ was analysed. The airline block time $\left(t_{B T}\right)$, the total amount of time a flight takes, i.e. from the time at which aircraft push back (contact bay)/taxi out (for non-contact bay) from the departure gate (Off-block), to the time at which aircraft arrive at destination arrival gate (On-block), vary for the same routes. Block time consists of the time to taxi-out from gate to the runway, holding time for departure, the actual flying time and the time to taxi to the arrival gate, but the published schedule for the flight does not spill up these elements apart. Block time can be calculated by taking the difference between Actual In block time and Actual off Block time and is given by

$$
t_{B T}=t_{A I}-t_{A O}
$$

The prediction depends on the various parameters at different phases of flight. This problem can be treated as a regression problem and the dependent variable is $t_{A I}$.

\section{Regression Tree-M5P Model}

The M5P is a non-linear regression model. The M5P (Wang \& Witten, 1996) is a reconstruction of Quinlan's M5 algorithm (Quinlan, 1992) for inducing trees of regression models. M5P is a combination of a conventional decision tree with the possibility of linear regression functions at each node. The M5P algorithm, the leaves are composed of multivariate linear models that build regression trees and which is one of the most commonly used classifiers of this family. The nodes of the tree are selected in such a way that, attributes maximise the expected error reduction as a function of the standard deviation of the output parameter.

M5P combined conventional decision tree based on the $t_{A T}$ values with linear regression functions at the nodes. The M5P regression tree model is given in Figure 2. This model uses six linear model equation and the equations vary with node, which depends on the departure time. So it is combination of linear model which varies with time. 
LMnum: 1

LMnum: 3

$$
\hat{t}_{A I}=1.06 * t_{A T}+102.73
$$

LMnum: 5

$$
\hat{t}_{A I}=0.99 * t_{A T}+120
$$

$$
\hat{t}_{A I}=0.89 * t_{A T}+222.2
$$

LMnum: 2

$$
\hat{t}_{A I}=0.98 * t_{A T}+117.70
$$

LMnum: 4

$$
\hat{t}_{A I}=1.07 * t_{A T}+41.86
$$

$$
\begin{aligned}
& \text { Mnum: } 6 \\
& \hat{t}_{A I}=0.99 * t_{A T}+119.59
\end{aligned}
$$

Multinomial logistic regression model to predict the shift in the schedule time of arrival

One of the key requirements for the Airport Operators and Airline Operators are the conformance predictability of scheduled flights arrival time with scheduled time. Prediction accuracy of the above hinges resource allocation and bay allocation. Here we propose a multinomial logistic regression model to predict the on time performance (Early, On time, and Delay) of the scheduled flights, based on schedule departure time variation (Early, On time and Delay) and Actual In block time $\left(\hat{t}_{A I}\right)$ predicted using M5PModel.

Multinomial logistic regression (Bohning, 1992)) is an extension of binary logistic regression, which uses multi-criteria discrete choice modeller, that allows classification between more than two categories. This is one of the most widely used model of machine learning, which embraces both binary and continuous variables of data, as is the case with this work. Therefore, the premises used in this model are much clearer than other methods such as discriminant function analysis (Starkweather \& Moske, 2011). The key assumption is that the options are independent. This assumption states that the probability of a choice depends on how many users select this option. This is nearly right in our situation, flight schedules are independent. The multinomial logistic regression model can be written as

$$
\begin{aligned}
& \operatorname{logit}(p)=\log (p /(1-p))=\beta_{0}+\beta_{1} * D E+\beta_{2} * D O+\beta 3 * D D+\beta_{4} * \\
& P_{A I S I}
\end{aligned}
$$

The logit has linear approximation relationship, and lot $=$ logarithm of the odds. $\mathrm{p}$ denotes probability, $P(Y=1), \mathrm{Y}$ is the response variable ie category of variation from scheduled time of arrival. Here the qualitative response variable has three categories namely Early, Delay, and On time. The explanatory variables DE, DO, DD are Early departure $\left(t_{A O}-t_{S O}<-5\right.$ minutes), On time Departure $\left(-5\right.$ Minutes $>t_{A O}-t_{S O}<10$ Minutes and Delayed Departure $\left(t_{A O}-t_{S O}>10\right.$ Minutes) respectively. $P_{\text {AISI }}$ is the difference between predicted Actual Inblock time using MLR model and scheduled arrival time. $\beta_{0}, \beta_{1}, \beta_{2}, \beta_{3}, \beta_{4}$ indicates 
refers to the effect of explanatory variable on the $\log$ odds that $\mathrm{Y}=1$. The data was split in to training set(75\%) and test set(25\%).

\section{Results and Discussion}

\section{Prediction of Actual Block Time Using Regression Tree - M5P Model}

The regression tree using M5P model is given in Figure 2. The model is able to predict the $t_{A I}$ using $t_{A T}$. In the Figure 2, on the leaf of the tree (inside box) denoted by LM (Linear Model) with the number of instances the test was carried out. The percentages shown in the leaf of the tree are the root relative squared error, other than LM5, this value is very small. In this model Actual time of Takeoff, less than 536.5 Minutes are represented by two linear models $L M 1$ and $L M 2$ based on the $t_{A T}$ values as given below,less than or equal to 214.5 Minutes and greater than 214.5 Minutes. Similarly, the departures above 536.5 Minutes were again classified in 5 models, as shown in the figure 2. On a detailed analysis of the LM equation, it was found that the intercept varies in each LM model. For example, consider $L M 1$ and $L M 2$

$$
\begin{gathered}
\hat{t}_{A I}=1.06 * t_{A T}+102.73 \\
\hat{t}_{A I}=0.98 * t_{A T}+117.70
\end{gathered}
$$

If $t_{A T}=0$, from $L M 1, \hat{t}_{A I} \approx 103$ Minute and from $L M 2 \hat{t}_{A I} \approx 118$ minutes this indicates that for different time interval the constant term varies. The coefficient of $t_{A T}$ in both case are comparable and almost equal and similar values of intercept can be seen for LM3 and LM6. The model gives better MAE and RMSE value for the same test set of MLR model. But the model doesn't consider the other attribute contribution for predicting the $t_{A I}$. In order to improve prediction accuracy we used MLR time varying model, which is being described in the next section.

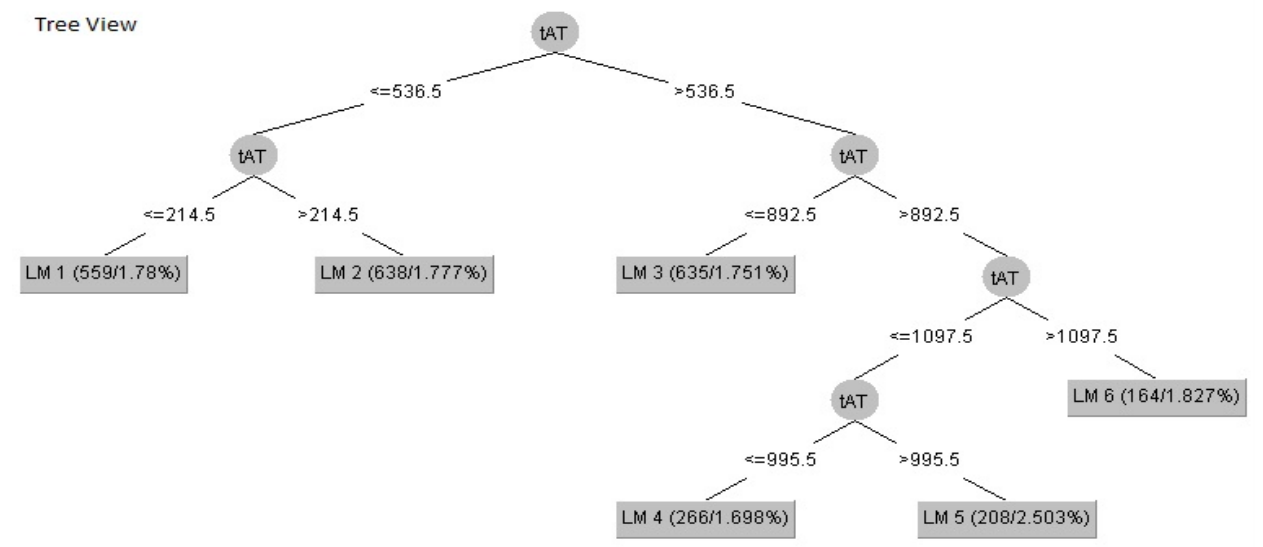

Figure 2. Regression Tree-M5P. 


\section{Varying Coefficient Models}

The desire of purely mathematical extension does not stimulate the varying coefficient models; instead, they come from the need in practice (Fan \& Zhang, 2008). Here we consider that some dynamic feature which may exist in the data set. We reconsidered the modelling strategy, used MLR model and explored the dynamic feature and made the model fit the data better. Altogether abandoning of the existing models will not be wise. It would probably be more sensible just to let the constant parameters evolve with specific characteristics, which leads to the varying coefficient models. In order to study variable coefficient, the data was split into 3-hrs set, and on that training and test set were created. It was observed from the previous section that the model parameter is varying with time, i.e. MLR model coefficient and intercept term will vary during the day and improvement in performance matrix were observed.

Table 1

MLR model with varying coefficients

\begin{tabular}{|c|c|c|c|c|}
\hline & \multicolumn{4}{|c|}{$\mathrm{t}_{\text {SO}}(\mathrm{Min})$} \\
\hline Coefficient & $0-180$ & $181-360$ & $361-540$ & $541-720$ \\
\hline Intercept & 103.85 & 109.79 & 101.49 & 135.83 \\
\hline ACT[T.A320] & -4.28 & - & - & - \\
\hline ACT[T.A32W] & -3.07 & 2.53 & 2.09 & -1.35 \\
\hline ACT[T.B38M] & -2.86 & - & - & - \\
\hline ACT[T.B738] & -5.67 & -1.35 & -1.56 & 3.13 \\
\hline RWY[T.Rwy11] & -2.81 & 2.39 & 0.6 & -4.54 \\
\hline RWY[T.Rwy27] & 2.74 & 1.84 & 2.57 & -2.06 \\
\hline RWY[T.Rwy28] & 5.33 & 2.88 & 8.17 & 0.55 \\
\hline RWY[T.Rwy29] & 1.57 & 1.33 & 0.58 & -4.08 \\
\hline SVC[T.Early] & 2.41 & 0.8 & -0.59 & -0.8 \\
\hline SVC[T.On Time] & 1.35 & 3.09 & 0.23 & 1.4 \\
\hline$t_{\text {AT }}$ & 1.06 & 0.99 & 1.01 & 0.97 \\
\hline
\end{tabular}

Table 1 shows the model intercept and coefficient variation at different time intervals. The first $12 \mathrm{hrs}$ data was taken for this analysis. The intercept value and coefficient values change for each model and prediction performance increased, and error reduced (shown in Table 1). 
Table 2

Performance matrix comparison

\begin{tabular}{|l|r|r|r|r|}
\hline & \multicolumn{4}{|c|}{ Test Data (Min) } \\
\hline & $\mathbf{0 - 1 8 0}$ & $\mathbf{1 8 1 - 3 6 0}$ & $\mathbf{3 6 1 - 5 4 0}$ & $\mathbf{5 4 1 - 7 2 0}$ \\
\hline Rsquare & 0.989 & 0.98 & 0.992 & 0.972 \\
\hline AdjRSquare & 0.988 & 0.979 & 0.992 & 0.97 \\
\hline MAE & 4.45 & 5.23 & 3.8 & 5.44 \\
\hline RMSE & 6.012 & 6.5 & 5.3 & 6.64 \\
\hline
\end{tabular}

It can be observed from Table 2 that the MSE and RMSE value is very good for this model. The minimum RMSE is 5.3, which indicate that 06:00 to 09:00 the model gives better prediction. A significant inference from this analysis is that static flying time is commonly used for expected landing time estimations in practise, although it varies over time.

\section{Improving Prediction Accuracy Using Exponential Moving Average of Flying Time}

We analysed improving prediction accuracy by adding the Moving Average value of historical flying time on input attributes of the MLR model. Moving averages tends to smooth out short-term irregularity in the data series based on an average of weighted observations. They are effective if the data series remains fairly steady over time. Further analysis was done on the data using Simple Moving average and Exponential Moving Average (EMA) of flying time. In Simple Moving average, since all the data points in the moving average process are given equal weight, this method fails to deal with non-stationary data. Exponential Moving Weighted Average methods are the techniques that place more weights on the recent observations. Holt (2004) proposed exponentially weighted moving averages (EMA) in dealing with forecasts of seasonal and trends. EMA's reaction directly depends on the pattern of the data. The EMA gives more weight to the recent values of flying time, and due to this feature, it can be a better model to capture the variation of the trend in a faster way. Here EMA of historical flying time of same type of aircraft is introduced in MLR model to improve the prediction accuracy. The exponentially weighted average of the forecast is an exponentially weighted (i.e. discounted) moving average with reducing factor $1-\alpha$ :

$$
F T_{(n)}=t_{A L(n)}-t_{A T(n)}
$$

Where $F T_{(n)}$ is the flying time of $n^{\text {th }}$ and $t_{A L(n)}, t_{A T(n)}$ presented are corresponding Actual landing time and Actual Take off time data. Here we have taken a window length of 5 . The revised model for predicting $t_{A I}$ is given by 
$\hat{t}_{A I}=-11.86+2.13 * A C T[T . A 320]+2.23 * A C T[T . A 32 W]+1.35 *$

ACT [T.B38M] $+1.38 * A C T[$ T.B738] $+1.73 *$ ACT [T.B739] $+-0.32 *$ $R W Y[T . R w y 11]+-0.42 * R W Y[T . R w y 27]+-0.17 * R W Y[T . R w y 28]+$ $-0.73 * R W Y[T . R w y 29]+0.70 * S V C[$ T.Early $]+0.50 *$ SVC [T. OnTime $]+$ $1.0 * t_{A T}+1.17 * E M A$

The performance matrix of the model is given in Table 3, Which clearly indicate that the proposed model gives excellent results for prediction of actual in block time comparing to the other model.

Table 3

Performance Matrix for prediction using EMA attribute

\begin{tabular}{|l|r|l|}
\hline & Training Data & Test Data \\
\hline Rsquare & $\mathbf{0 . 9 9 9}$ & - \\
\hline AdjRSquare & $\mathbf{0 . 9 9 9}$ & - \\
\hline MAE & $\mathbf{3 . 8 0}$ & $\mathbf{3 . 6 3}$ \\
\hline RMSE & $\mathbf{4 . 9 2 2}$ & $\mathbf{4 . 6 5}$ \\
\hline
\end{tabular}

The model is able to predict the Gate in time $\left(\hat{t}_{A I}\right)$ for various type of aircraft at the time of departure. The residual plot of the same is given in figure 3. The residual plot shows that distribution is normal and the mean is approximately zero, which indicate that the data follows MLR model and output can be predicted using MLR model.

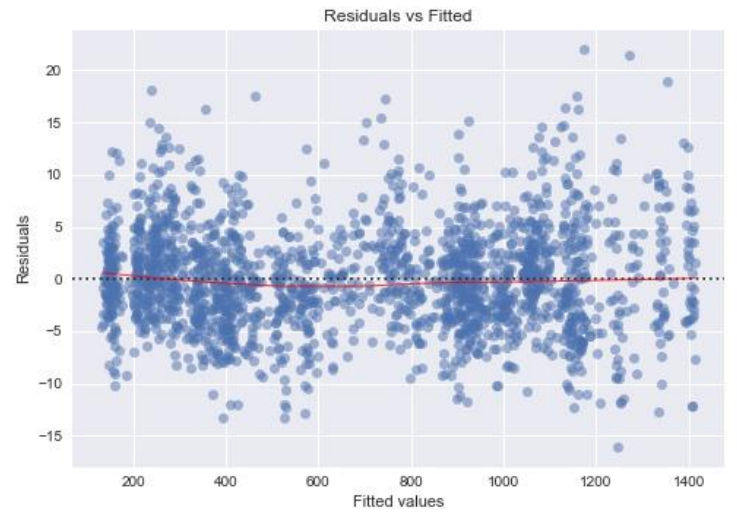

Figure 3. Residual vs Fitted Values using EMA Attributes. 


\section{Predicting Scheduled Arrival Time Variation Using Logistic Regression}

The test results show that proposed mode correctly classified 434 instances out of 522 test instances with accuracy of $83.14 \%$. The confusion matrix is given in table 4 . The $\beta$ parameter for each explanatory variable and intercept is given in table 5. The Precision for Delay, early and On time classification is $0.909,0.864,0.725$ respectively. The classification results were compared with the on time prediction proposed by (Etani, 2019)) and the proposed model gives better prediction results with minimal complexity, minimal attribute and computational cost. Precise schedule deviation prediction can be used to allocate airline resources efficiently, reduce turnaround times, minimise unnecessary gate changes and predict delays in departure of succeeding leg. In the case of a ground delay program in ATFM measures, arrival delay is a key element for on-time performance (Etani, 2019).

Table 4

Confusion Matrix

\begin{tabular}{|r|r|c|l|}
\hline \multicolumn{1}{|c|}{$\mathrm{a}$} & \multicolumn{1}{c|}{$\mathrm{b}$} & $\mathrm{c}$ & Classified as \\
\hline 159 & 1 & 17 & $\mathrm{a}=$ Delay \\
\hline 0 & 146 & 32 & $\mathrm{~b}=$ Early \\
\hline 16 & 22 & 129 & $\mathrm{c}=$ OnTime \\
\hline
\end{tabular}

Table 5

Logistic Regression Coefficients

\begin{tabular}{|c|r|r|}
\hline Variable & \multicolumn{1}{|l|}{ Delay } & \multicolumn{1}{l|}{ Early } \\
\hline$\beta_{4}$ & 0.3115 & -0.3399 \\
\hline$\beta_{3}$ & -0.3077 & -0.2133 \\
\hline$\beta_{2}$ & -0.1214 & 0.0762 \\
\hline$\beta_{1}$ & 0.886 & 0.2626 \\
\hline$\beta_{0}$ & -3.0891 & -1.7765 \\
\hline
\end{tabular}

\section{Conclusion}

The findings of the study are in line with our initial experience-based observations that airlines prefer to 'pad' (add additional time) flight schedules to give the appearance that most flights arrive on time. Results of the study indicate that airlines push back /taxi early from the departure gate with the advantage of comparatively less average flying time, which happens early in the morning and late in the evening. This may be one of the reasons because of which pilots are attempting to leave earlier than their scheduled departure time. The study has given an insight in to the huge variation in actual block time from schedule block 
time, which varies in a large window from the mean value of the block time for aircraft operated between the same departure destinations. The inability of existing flight time calculation method in tracing temporal variation in flight time will lead to a significant gap between the actual flight time and the expected flight time, which has a greater effect than the early departure of the scheduled flights. This is significant finding of the research to improve calculation of estimated flying time.

For improving predictability, a non-linear (M5P) model was analysed. As this model varies at different nodes, it shows that time-varying Linear model can improve prediction accuracy. The time-varying attribute model was used to capture the dynamic changes in the data. This model gives better performance matrix as compared to the $M 5 P$ model. Finally, by using the additional attribute, an exponential moving average of previous flying time, excellent predictability was obtained. Combination of MLR and Logistic regression model provides better predictability of variation in scheduled time of arrival and airline on time performance at the time of departure. The proposed model is able to provide delay information of trailing flight with fair amount of accuracy which can be used to improve ATFM GDP. The future works include the calculation of departure time or off block time of succeeding flight using machine learning technique for Ground Delay programs for multiple airports. Thereby more accurately arrival sequence can be predicted and ATFM ground delay program can be implemented more efficiently.

\section{Recommendations}

The research recommends that the existing method used in ATM/ATFM should be improvised for predicting the expected time of arrival. Early departures lead to major flying time differences and some of the scheduled flights benefited from the same, but this should be minimised or eliminated. Nonlinear (M5P) and time varying models of regression method gives better predictability of gate in time, even if flying time varies significantly. A key feature in the model based on machine learning is the exponential moving average of previous flying time, which also enhances the predictability of on time arrival. 


\section{References}

Bohning, D. (1992). Multinomial logistic regression algorithm. Annals of the Institute of Statistical Mathematics, 44(1), 197-200.

Choi, S., Kim, Y. J., Briceno, S., \& Mavris, D. (2016). Prediction of weatherinduced airline delays based on machine learning algorithms. In 2016 IEEE/AIAA 35th digital avionics systems conference (dasc) (pp. 1-6).

Choi, S., Kim, Y. J., Briceno, S., \& Mavris, D. (2017). Cost-sensitive prediction of airline delays using machine learning. In2017 IEEE/AIAA 36th digital avionics systems conference (dasc) (pp. 1-8).

Dahiru, T. (2008). P-value, a true test of statistical significance? A cautionary note. Annals of Ibadan Postgraduate Medicine, 6(1), 21-26.

Deepudev.S, V., Palanisamy.P, \& Manjunath.K.Nell. (2020). A machine learning based approach for prediction of actual landing time of scheduled flights. In Proceedings of International Conference on Recent Trends in Machine Learning, IoT, Smart Cities and Applications, Advances in Intelligent Systems and Computing, Springer, 1245, 755-766.

Delhi Airport. (2020). Fact sheet. Retrieved from www.newdelhiairport.in/ medias/factsheet

Ding, Y. (2017). Predicting flight delay based on multiple linear regression. In Iop conference series: Earth and Environmental Science, 81, 012198.

Donohue, G., Zellweger, A., Rediess, H., \& Pusch, C. (2001). Air transportation system engineering: progress in astronautics and aeronautics. American Institute of Aeronautics and Astronautics.

Etani, N. (2019). Development of a predictive model for on-time arrival flight of airliner by discovering correlation between flight and weather data. Journal of Big Data, 6(1), 85.

Evans, A. D., Lee, P., \& Sridhar, B. (2018). Predicting the operational acceptance of airborne flight reroute requests using data mining. Transportation Research Part C: Emerging Technologies, 96, 270-289.

Fan, J., \& Zhang, W. (2008). Statistical methods with varying coefficient models. Statistics and its Interface, 1(1), 179.

Fleurquin, P., Arranz, A., Etxebarria, I., Ciruelos, C., Eguíluz, V. M., \& Ramasco, J. J. (2016). Comparing the modelling of delay propagation in the US and European air traffic networks. Journal of Air Transport Management, 56, $12-18$.

Glina, Y., Jordan, R., \& Ishutkina, M. (2012). A tree-based ensemble method for the prediction and uncertainty quantification of aircraft landing times. In American meteorological society-10th conference on artificial intelligence applications to environmental science, New Orleans, LA. 
Hof, H. J. (2005). Development of a performance framework in support of the operational concept. In ICAO mid region global atm operational concept training seminar.

Hoffman, R., Mukherjee, A., \& Vossen, T. (2011). Air traffic flow management. quantitative problem solving methods in the airline industry: A modelling methodology handbook. In C Barnhart \& B. Smith (Eds.), International Series in Operations Research and Management Science. Norwell, MA: Springer.

Holt, C. C. (2004). Forecasting seasonal and trends by exponentially weighted moving averages. International Journal of Forecasting, 20(1), 5-10.

International Air Transport Association. (2018). India's air transport sector. www.iata.org/contentassets/eec5052bac6a4fd68f98e751b0b97d21/indiaaviation-summit-aug18.pdf

Kern, C. S., de Medeiros, I. P., \& Yoneyama, T. (2015). Data-driven aircraft estimated time of arrival prediction. In 2015 annual IEEE systems conference (syscon) proceedings (pp. 727-733).

Kim, M. S. (2016). Analysis of short-term forecasting for flight arrival time. Journal of Air Transport Management, 52, 35-41.

Kuhn, K. D. (2016). A methodology for identifying similar days in air traffic flow management initiative planning. Transportation Research Part C:

Emerging Technologies, 69, 1-15.

Morisset, T., \& Odoni, A. (2011). Capacity, delay, and schedule reliability at major airports in Europe and the United States. Transportation Research Record, 2214(1), 85-93.

Prasad, A. S., \& Chauhan, P. A. (2018). Data mining predictive analysis on airlines performance. International Journal of Pure Applied Mathematics, $118,1-12$.

Quinlan, J. (1992). Learning with continuous classes v: 5th Australian joint conference on artificial intelligence. A. Adams a. \& L. Sterling (Eds.). Hobart, Tasmania: World Scientific.

Rapajic, J. (2009). Beyond airline disruptions. New York: Ashgate Publishing.

Rapajic, J. (2018). Beyond airline disruptions: Thinking and managing anew. New York: Routledge.

Rebollo, J. J., \& Balakrishnan, H. (2014). Characterization and prediction of air traffic delays. Transportation Research part C: Emerging Technologies, 44, 231-241.

Starkweather, J., \& Moske, A. K. (2011). Multinomial logistic regression. Retrieved http://www. unt.edu/rss/class/Jon/Benchmarks/ MLR_JDS_Aug2011.pdf,29, 2825-2830. 
Takeichi, N. (2017). Nominal flight time optimization for arrival time scheduling through estimation/resolution of delay accumulation. Transportation Research Part C: Emerging Technologies, 77, 433-443.

Thiagarajan, B., Srinivasan, L., Sharma, A. V., Sreekanthan, D., \& Vijayaraghavan, V. (2017). A machine learning approach for prediction of on-time performance offlights. In2017 IEEE/AIAA 36th digital avionics systems conference (dasc) (pp.1-6).

Wang, Y., \& Witten, I. H. (1996). Induction of model trees for predicting continuous classes. Retrieved from https://www.researchgate.net/publication/33051395_Induction_of_model_ trees_for_predicting_continuous_classes

Williams, J. K. (2014). Using random forests to diagnose aviation turbulence. Machine Learning, 95(1), 51-70.

$\mathrm{Wu}$, C.-L. (2005). Inherent delays and operational reliability of airline schedules. Journal of Air Transport Management, 11(4), 273-282 